\title{
Percepción de habitantes del Norte de Santander sobre limitaciones del buen gobierno*
}

\author{
Albornoz Arias, Neida Coromoto** \\ Mazuera Arias, Rina***
}

\section{Resumen}

El presente artículo analiza la percepción de habitantes del Norte de Santander (Colombia) sobre limitaciones del buen gobierno. Se consideran dos elementos del nuevo paradigma de gestión pública, propuestos por el Fondo de las Naciones Unidas para el Desarrollo y el Banco Mundial: ausencia de corrupción y confianza en las instituciones públicas (de todas las ramas del poder, en los ámbitos nacional, departamental y municipal). La percepción de los ciudadanos se obtuvo a través de la aplicación de una encuesta a una muestra para poblaciones finitas de 2.398 personas con edades entre 18 y 69 años, en los cuarenta municipios del Departamento. Existe una marcada percepción de corrupción y desconfianza en las instituciones públicas del Norte de Santander. Esto incide en la credibilidad ciudadana y trae consigo carencias para la existencia de un buen gobierno. En este sentido es fundamental la definición de estrategias para fomentar la participación ciudadana, acompañadas de políticas anticorrupción para la existencia de un buen gobierno.

Palabras clave: Buen gobierno; corrupción; confianza; participación ciudadana; democracia.

Recibido: 10-05-16. Aceptado: 30-07-16

* Artículo de resultados enmarcado en el proyecto de investigación: Análisis del contrabando en el Departamento Norte de Santander. Un enfoque desde la institucionalidad; de la Universidad Simón Bolívar - sede Cúcuta, Grupo de investigación Altos Estudios de Frontera -ALEF-. Fecha de inicio: 23 de enero de 2015. Fecha de finalización: 31 de mayo de 2016. Financiado por la Universidad Simón Bolívar - sede Cúcuta.

** Contador Público Universidad Católica del Táchira (UCAT). Doctora en Ciencias Sociales y Jurídicas (Universidad de Córdoba - España). Docente e investigadora de la Universidad Simón Bolívar (Cúcuta) Colombia y UCAT. e:mail: n.albornoz@unisimonbolivar.edu.co; albornoz@ucat.edu.ve

*** Abogada Universidad Católica del Táchira (UCAT). Doctora en Derecho en el programa Derecho de Familia y de la Persona (Universidad de Zaragoza - España). Docente e investigadora de la Universidad Simón Bolívar (Cúcuta) Colombia y UCAT. Decana de investigación y Postgrado de la UCAT. e:mail: r.mazuera@unisimonbolivar.edu.co; rmazuera@ucat.edu.ve 


\title{
Perception of Inhabitants of Norte de Santander on Limitations of Good Governance
}

\begin{abstract}
This article analyzes the perception of inhabitants of Norte de Santander (Colombia) on limitations of good governance. Two elements of the new public management, proposed by Fund of the United Nations Development Programme (PNUD) and the World Bank are considered: the absence of corruption and trust in public institutions (of all branches of power at the national, departmental and municipal areas). The perception of citizens was obtained through the application of a survey to a sample of 2,398 people between the ages of 18 and 69 years old, in forty municipalities of the department. The results demonstrated the perception of corruption and mistrust in public institutions which demand strategies to encourage citizen participation and anticorruption policies for the existence of good government.
\end{abstract}

Keywords: Good government; corruption; trust; citizen participation; democracy.

\section{Introducción}

La conformación de los Estados modernos reposa en la disposición y valor de la probidad en el comportamiento de los servidores públicos. Para su sostenimiento, las democracias en el mundo requieren moralidad para cumplir los preceptos en los cuales se sustenta su legitimidad ante la sociedad, explorando nuevas y mejores estrategias para conciliar y satisfacer las exigencias de una sociedad urgida de resultados en cuanto a la calidad de las acciones y del uso de los recursos públicos.

Es así como nace el buen gobierno como un paradigma de la Nueva Gestión Pública (NGP), como una alternativa que pretende resguardar el uso de los recursos públicos y mostrar ante las sociedades, mecanismos que le permitan reducir la brecha de desconfianza y recuperar los principios centrales del Estado democrático, como son la ética y rendición de cuentas. Se asume como buen gobierno la práctica que conduce a un gobierno efi- ciente, con servicios de calidad, programas eficaces, transparencia gubernamental, ampliación de la libertad de los gerentes para realizar su gestión y mejora de la imagen de líderes políticos (Pollit y Bouckaert, 2000).

Ahora bien, para ahondar en los orígenes del buen gobierno es preciso revisar lo descrito por Sánchez (2015), citando los aportes de Cortina al referirse a lo expuesto hace 2500 años por Confucio, en cuanto a los grandes valores y la estructura fundamental del buen gobierno. Esta inferencia de Confucio la basó en la ética, un principio que todo gobernante debe practicar en su accionar, que debe regir sus quehaceres y por ende deben ser la razón de su compromiso como estadista y como servidor público en beneficio de su sociedad.

Por su parte, la idea de buen gobierno como fin del Estado según Bobbio citado por Aguilar (2000), aparece desde el cambio de pensamiento político en el siglo XIII, junto con la finalidad de conservar la justicia, entendiéndose como la 
realización del derecho establecido; mientras que el buen gobierno involucra al bien común en todas sus formas, no solo para la custodia del derecho existente, sino también por la creación legislativa de derecho, a fin de cumplir de forma concreta con la misión del bien común.

Ya en la era global, el concepto de buen gobierno y probidad en los agentes públicos propician la interacción entre los gobiernos y los ciudadanos, la cual implica "imperativos éticos" y de "transparencia" para el Estado, que inspiran la confianza en esa interacción (Aranda, 2013: 217). Un gobierno no transparente genera fractura social y desconfianza (Tornos et al, 2012).

Los antes referido obliga a investigar sobre la existencia del buen gobierno en el Norte de Santander (Colombia); departamento fronterizo, que por su ubicación geográfica colindante con Venezuela y distanciado de la capital, requiere de la existencia de un buen gobierno que implique la participación ciudadana para fortalecer la democracia.

Como premisas del buen gobierno, se abordan los principios ${ }^{1}$ : ausencia de corrupción y confianza, ambos coincidentes con los elementos teóricos de la institucionalidad, considerados en el trabajo de investigación "Análisis del contraban- do en el Norte de Santander, desde la percepción de los constructos de la institucionalidad" de Albornoz et al (2016). Interesa el contexto del Departamento Norte de Santander (Colombia), porque la Universidad Simón Bolívar (sede Cúcuta), contribuye desde su grupo de investigación Altos Estudios de Frontera (ALEF), con la generación de conocimiento y propuestas en pro desarrollo de la frontera colombo-venezolana.

El reto es el fortalecimiento del gobierno local, de su capacidad de transición hacia el desarrollo a través de políticas que reduzca la corrupción, aumente la transparencia y confianza, ampliando las capacidades humanas, que incida en la percepción de los ciudadanos sobre la democracia (Ciborra y Navarra, 2005; Carbone, 2010; Panday y Rabbani, 2011; Brower, 2015).

El objetivo del presente artículo es analizar la percepción ${ }^{2}$ de habitantes del Norte de Santander (Colombia) sobre limitaciones del buen gobierno. Se consideran dos elementos de la nueva gestión pública, propuestos por el Fondo de las Naciones Unidas para el Desarrollo y el Banco Mundial: ausencia de corrupción y confianza en las instituciones públicas colombianas (de todas las ramas del poder público en los ámbitos nacional, de-

1 En el próximo apartado se exponen los principios en los cuales se basa un buen gobierno, propuestos por el Programa de las Naciones Unidas para el Desarrollo (UNDP, 1997).

2 "La percepción hace referencia a algo captado en el contexto de investigación a partir de lo cual se construyen imaginarios que suponen la base para la proyección de normas, valores y principios acordados socialmente. En este sentido, el contexto social, político y económico serán decisivos para que una comunidad de individuos capten e interpreten la realidad en función de sus experiencias de vida" (Albornoz, et al, 2016: 14). 
partamental y municipal) y su incidencia en la democracia.

Ambos elementos se materializan en la responsabilidad, transparencia, eficacia y eficiencia del Estado; considerando que la transparencia ayuda a combatir la corrupción (Campos y Peña, 2015; UNDP, 1997; The World Justice Project, 2014). Sin embargo, en la práctica el buen gobierno tiene limitaciones provenientes de la existencia de corrupción, que genera desconfianza de los ciudadanos hacia el sector público; tal como lo revela el Índice de Percepción de la corrupción del sector público, que en Colombia fue de $37^{3}$ en 2014, ubicando a Colombia en el puesto 94 de 175 países evaluados (Transparency International, 2014).

La población consultada estuvo conformada por hombres y mujeres entre el grupo etario de 18 a 69 años, con residencia en el Departamento. La información fue recopilada para el estudio en los cuarenta municipios del Departamento, durante el período $1^{\circ}$ de abril hasta el 30 de junio de 2015. Fue determinada una muestra para poblaciones finitas. Se empleó un nivel de confianza del $95 \%$, con un error máximo del $2 \%$. El tamaño de la muestra fue 2.394 individuos, discriminados según edad y sexo. Se empleó un muestreo aleatorio simple estratificado con afijación proporcional (Albornoz, et al, 2016).

El instrumento de recolección de información fue una encuesta semies- tructurada. Los resultados provienen de las tablas de contingencia que permiten realizar el análisis descriptivo de la situación.

\section{El buen gobierno}

La noción de buen gobierno (good governance) surge formalmente desde inicios de los años noventa como un paradigma de la Nueva Gestión Pública (NGP) y como resultado de un estudio del Banco Mundial, relacionado con la crisis y crecimiento que mantenía África Subhariana, cuyos países adoptaron medidas que les permitió experimentar un crecimiento que favoreció sus balances, aplicación de política sana, coyuntura favorable e incremento de auxilios financieros extranjeros para aliviar la deuda externa.

Esto, demandó una NGP para un buen gobierno, caracterizada por ser eficiente, transparente, con servicios públicos de calidad, programas eficaces y mejora de la imagen de líderes políticos. Desde entonces, el concepto de buen gobierno se ha divulgado en los estudios sobre desarrollo, por su importancia especialmente para países emergentes (Pollit y Bouckaert, 2000, Carbone, 2010; Doornbos, 2001; World Bank, 1992; Weiss, 2000; Woods, 2000). Un buen gobierno es responsable y los poderes públicos rinden cuentas, son transparentes, los funcionarios públicos son previsivos y

3 La escala va de 0 (percepción de altos niveles de corrupción) a 100 (percepción de bajos niveles de corrupción). Una alta puntuación evidencia un contexto donde ocurre el soborno, actos de corrupción impunes y las instituciones públicas no dan respuesta a las necesidades de los ciudadanos. 
existe un Estado de Derecho (CLAD, 2006; Caddy y Vergez, 2003).

En el marco de la NGP, el Programa de las Naciones Unidas para el Desarrollo - PNUD (UNDP, 1997), propuso un estilo de dirección enfocado en la gobernabilidad como eficiencia del gobierno y del Estado para formular y ejecutar políticas económicas y administrar la gestión pública; y articula el concepto de buen gobierno con cinco principios clave del desarrollo humano y se propone que los mismos tengan universalidad. Estos se relacionan con el ejercicio de la autoridad económica, administrativa y política, para que así el Estado, fomente la cohesión social y bienestar de la población, lo cual requiere de la participación ciudadana en la gestión pública, como un nuevo paradigma social que busca la democracia plena (Montecinos, 2012; CLAD, 2009; Morales, Nuñez y Delfin, 2006).

Los principios antes indicados son a) legitimidad y voz: comprende la participación, la libertad de asociación y de expresión y la orientación hacia el consenso; b) dirección: los líderes (gobierno) y los ciudadanos, tienen una perspectiva amplia y de largo plazo en el buen gobierno y el desarrollo humano, basada en la confianza; c) rendimiento: la capacidad de respuesta de manera eficaz y eficiente de las instituciones y sus procesos; d) responsabilidad: comprende la rendición de cuentas y la transparencia que se refiere al libre flujo de información sobre los procesos en las instituciones; d) justicia: involucra la equidad y Estado de Derecho (existencia de marcos legales justos y aplicables de manera imparcial, en particular las leyes sobre los derechos humanos) (UNDP, 1997).
EI PNUD (2014) concibe que las prioridades socioeconómicas y políticas, se fundamentan en un consenso social, un buen gobierno implica la buena actuación de las instituciones gubernamentales, gestión de sectores públicos y privados, descentralización y articulación con organizaciones de la sociedad civil. Además, tiene en cuenta factores culturales, características nacionales, liderazgos, responsabilidad política, procesos de participación y construcción de capacidades (PNUD 2011; UNDP, 1995; Weiss, 2000; UNDP, 2014); y para lograrlo, promueve "instituciones formales (normas y reglamentos) e informales (lógica de lo apropiado en cada organización pública)" (Villoria, 2013: 10).

Para que pueda materializarse la promoción de normas formales e informales, coexiste un contrato social entre gobernantes y gobernados que encarna la entrega efectiva de bienes-políticos a los Estados-nación para que sean administrados de manera efectiva y en nombre de sus habitantes, bajo la base de la confianza y creencias sociales.

En este sentido, una nación con buen gobierno posee Estado de Derecho; las libertades políticas y civiles; estabilidad macroeconómica; atención médica y sanitaria; educación; carreteras, vías férreas, las arterias de comercio; redes de comunicaciones; un contexto económico e institucional dentro del cual los ciudadanos pueden prosperar; apoyo a la sociedad civil; y un método para regular la distribución de los bienes ambientales comunes, con una democracia consolidada, participativa y con calidad institucional (Besançon, 2003; Bonifacio, 2006; Grindle, 2007). 
También es necesario que existan instituciones democráticas de calidad, la mejora de los estándares de gobierno (Bhagwati y Gambari, 2005) y la creación de una sector privado productivo (Nanda, 2006; Nonnel, 2006; Oppenheimer, 2005). No obstante, la preocupación de académicos ha sido el desarrollo de una mejor comprensión de las diferentes formas en que las relaciones de poder y autoridad, se estructuran en diferentes contextos, así como los modos de interrelación entre entre el Estado y sociedad civil (Hyden y Bratton, 1995; Leftwich, 1994; Tordoff, 2002).

Un buen gobierno aporta capacidades para evitar toda dominación arbitraria sobre las personas y permite desarrollar un modelo de vida (Villoria e Izquierdo, 2015). Otro aporte para entender el significado de buen gobierno, es el destacado por Pezo (2012), quien sostiene que el término buen gobierno no solo se refiere a la forma en que el poder político es ejercido en un país, sino que también refleja la capacidad de los gobiernos, de sus instituciones y sus políticas para canalizar y solucionar los conflictos que surgen en el seno de la sociedad mediante reglas y procedimientos democráticos. La participación ciudadana es uno de sus principales ejes, involucrando la dimensión administrativa-gerencial del gobierno; que requiere un examen de las labores cotidianas de la instituciones (Woods, 2000; Doornbos, 2001; Doornbos, 2006).

Aunado a ello, el buen gobierno requiere empresas interdependientes y complementarias que permiten impugnar al régimen autoritario, la asignación eficiente de los recursos públicos, el consenso entendido como la voluntad políti- ca de la sociedad, la cual se traduce en la legalidad de las acciones administrativas y las políticas del Estado como fuente de legitimidad (Aguilar, 2000).

La Escuela Superior de Administración Pública de Colombia (ESAP, 2007) señala que un Código de Buen Gobierno son las disposiciones voluntarias de autorregulación de quienes ejercen el gobierno de las entidades, que a manera de compromiso ético buscan garantizar una gestión eficiente, íntegra y transparente en la administración pública.

Hoy en día, se entiende la ausencia de un buen gobierno como: a) falta de responsabilidad y rendición de cuentas de los poderes públicos, b) falta de transparencia, c) escasa capacidad de previsión de los políticos y funcionarios, y d) ausencia de Estado de Derecho (Villoria, 2013). Uno de los mayores obstáculos para la existencia de un buen gobierno es la creciente corrupción en el manejo de los bienes del Estado (Gómez, 2006), por la existencia de espacios públicos en la que se le oculta información a los ciudadanos, sobre todo en tiempos en que los recursos son escasos y la exigencia de conocer en qué se invierten los fondos públicos (Soriano, 2014).

Para solventar estos escollos y cumplir los principios que rigen el concepto de buen gobierno y la administración pública, Larach (2015: 263) señala que deben existir los siguientes elementos: apertura, amplitud de las instituciones en su funcionamiento, comunicación, toma de decisiones y uso del lenguaje; participación, la cual se describe a través de la efectiva intervención de los ciudadanos en las fases de formulación y ejecución de las políticas públicas con el fin de fortalecer 
las instituciones y la confianza de los ciudadanos en esta; la responsabilidad para asumir las consecuencias de cada actuación institucional; eficacia en la producción de los resultados perseguidos con idoneidad, efectividad y proporcionalidad y coherencia que debe estar presente en las políticas y acciones bajo un buen liderazgo y compromiso institucional.

Entre las necesidades de fortalecimiento institucional, Diego (2006) reseña que la Organización para la Cooperación y Desarrollo Económico (OCDE) comenzó a diseñar una infraestructura compuesta de tres pilares: 1) control con un marco legal, con mecanismos adecuados de responsabilidad y participación y escrutinios políticos; 2) orientación y compromiso de los líderes políticos, códigos de conducta y mecanismos de socialización profesional como la educación y la formación; y 3) gestión, con condiciones sólidas de servicio público, coordinación de esta infraestructura, de este departamento u organismo central con competencia en materia de ética.

En Colombia, el gobierno nacional promociona a través de sus organismos, las prácticas y acciones para un buen gobierno, a fin de alcanzar la prosperidad democrática y el fortalecimiento institucional. En este sentido, el Departamento Nacional de Planeación (DPN, 2016) establece 5 principios que orientan el esfuerzo de este organismo en función de cumplir las premisas del buen gobierno: 1) la transparencia y la rendición de cuentas; 2 ) una gestión pública efectiva; 3) la participación y servicio al ciudadano; 4) la vocación por el servicio público; y 5) estrategias para la lucha contra la corrupción.
En el caso del Departamento Norte de Santander, el gobierno departamental, promulgó el Código de Buen Gobierno para esta entidad regional. En el mismo, se declaran las políticas del modelo gerencial para el control interno que deberá regir la gestión, así como también las orientaciones a seguir por parte de los funcionarios públicos a fin de cumplir con los objetivos que persigue la gestión departamental en la función social que cumple (Gobernación Norte de Santander, Decreto $N^{\circ}$ 000427).

Este Código evidencia en Norte de Santander, un acercamiento a la adopción de principios de buen gobierno. No obstante, la fuerza de la universalidad de los mismos dependerá de la responsabilidad y transparencia del gobierno frente a sus gobernados, la libertad y participación ciudadana, eficiencia y eficacia de las instituciones públicas y la existencia de un Estado de Derecho que respalde los deberes y obligaciones del Estado y los ciudadanos (Brower, 2015; Carbone, 2010; Ciborra y Navarra, 2005; Panday y Rabbani, 2011).

\section{3. ¿Ausencia de corrupción?}

La corrupción consiste en el abuso de confianza o autoridad para el beneficio privado, puede darse en el sector público o privado, no es exclusivo de funcionarios públicos sino también de particulares que ocupan cargos de confianza (Secretaría, 2005). Desde una visión socio-antropológica, la corrupción produce una división social debido a las condiciones existentes de exclusión social, donde un grupo tiene acceso a las decisiones políticas o recursos y otro persuade a la autoridad 
para obtener lo que desea debido a relaciones personales, familiares existentes o al soborno. La desigualdad entre los grupos sociales menoscaba la democracia. La corrupción es una manera de influir y persuadir a quienes ejercen la autoridad y de esa manera se relacionan con el Estado (Carassale, 2013)

La corrupción puede ser centralizada, el gobierno organiza la corrupción desde el más alto nivel, o descentralizada, las personas con poder tratan de extender sus favores corruptos. Puede darse en el nivel político, administrativo, judicial y en el sector privado. En cualquiera de los casos para su verificación se requiere de unos acuerdos secretos no claros al margen de la ley. Acuerdos corruptos cuyo cumplimiento se garantizan con mecanismos alternos a la justicia como: "el uso de rehenes, de intermediarios, de violencia, la integración vertical, la reputación, la repetición y el uso de redes sociales" (Boehm y Graf, 2009: 52).

Existen diversas causas que pueden influir en la corrupción entre ellas pueden mencionarse: los determinantes políticos, el régimen legal y judicial, las instituciones fiscalizadoras, el entorno económico, regulatorio y de finanzas públicas, la administración del sector público, el nivel de desarrollo del país (Kaufmann, 2014; Secretaría, 2005). No obstante, en relación a la última causa, diversas investigaciones han señalado que puede coexistir crecimiento y desarrollo económico en Estados cuyos gobiernos tengan prácticas corruptas (Sandoval, 2016).

De los diversos factores socio-políticos, jurídico-institucionales, económicos y ético-culturales que determinan la existencia y propagación de la corrupción (Treisman, 2000), interesa resaltar las dos primeras categorías.

Entre los factores socio-políticos como los partidos políticos, el sistema de gobierno y el régimen político, se parte de la relación que existe entre democracia y corrupción, pues es el sistema político más idóneo para la lucha contra la corrupción. Los partidos políticos tiene un rol indispensable en la democracia, sin embargo, existen algunas características de los mismos que propician la corrupción, como lo son: el clientelismo político; el monopolio partidista; el costo de la vida política; la debilidad de la noción de legalidad y autoridad, cada vez que los líderes políticos incurren en conductas corruptas por violación a la ley sin existir un castigo, se crea una desconfianza en los ciudadanos debilitándose el respeto por las autoridades y las instituciones que represen$\tan$ (Orrego, 2014).

En el régimen político puede dar lugar a la corrupción las debilidades de la democracia, como la falta de participación ciudadana en el control de actos corruptos que exijan combatir la misma y sus efectos, las personas esperan cualquier cosa del Estado, no se conoce cuáles son los medios propicios para organizarse y participar en los diversos asuntos de la administración pública y la poca independencia de los poderes públicos. Lo indicado guarda relación estrecha con los factores institucionales, entre los cuales puede mencionarse el crecimiento desproporcionado del poder ejecutivo en comparación con las otras ramas del poder, donde juega un rol importante el poder judicial, pues tiene una doble relación en la corrupción como causa y conse- 
cuencia por la debilidad del poder judicial dada por la falta de independencia ante el poder ejecutivo, falta de recursos y su ineficacia (Orrego, 2014).

El poder legislativo puede ayudar a la corrupción por la poca capacidad técnica para controlar efectivamente el poder ejecutivo, limitaciones constitucionales o legales para investigar los actos del gobierno, y la falta de control interno dentro del poder legislativo, lo que da lugar a su poca credibilidad y la percepción de ineficacia y corrupción por parte de la población (Orrego, 2014); percepción detectada en la investigación realizada en Norte de Santander, donde el $62,7 \%$ de los encuestados, manifestaron que el Congreso de la República en Colombia, es un órgano con mucha corrupción en comparación con los demás instancias del poder público y el $24,9 \%$ consideran que tiene algo de corrupción.

La debilidad de los órganos de control internos y externos, dada por la desactualización y excesiva legalidad de los mismos, limitándose a los mecanismos legales deja por fuera algunos actos de corrupción y la vigilancia de los resultados relacionados con la eficacia y eficiencia del gasto público; la insuficiencia de recursos humanos y económicos; y la falta de independencia de los órganos de inspección son parte de los factores institucionales que facilitan la corrupción (Orrego, 2014). En el estudio, la percepción del $43,9 \%$ de los encuestados es que existe mucha corrupción en la Contraloría, y algo de corrupción para el $36,7 \%$.

La corrupción incide en la separación de poderes obstaculizándose el cumplimiento efectivo de los fines del Estado, se resiente la democracia, atentan- do contra los derechos humanos al poder derogarse de facto los mismos, aun cuando siguen garantizados en la carta magna; la corrupción es un problema político por estar unida al sector público e influir en la actuación pública, con gran incidencia social (Marín, 2004; García, 2000).

De manera directa o indirecta los más afectados, son los pobres y los más vulnerables (Boehm y Graf, 2009). Incidencia que está más marcada cuando proviene de la principal autoridad administrativa en el ámbito nacional, departamental y municipal; problema que se percibe en la investigación cuando los encuestados manifestaron que existe mucha corrupción en el gobierno nacional $(57,9 \%)$, gobierno departamental $(55,8 \%)$ y gobierno municipal $(54,9 \%)$, o algo de corrupción en el gobierno departamental $(32,2 \%)$, gobierno municipal $(32 \%)$ y gobierno nacional $(29,6 \%)$.

Las víctimas de la corrupción pueden ser varias: la sociedad, la administración pública, la economía pública; debido a que distintos sujetos públicos y privados logran verse afectados por el favoritismo corruptivo (Mannozzi, 2009; Mauro, 1995); se afecta de manera directa los procesos democráticos (Warren, 2005), disminuyendo la confianza de los ciudadanos en los distintos poderes públicos (Diego, 2012).

La corrupción es un problema que existe en el Norte de Santander de acuerdo a la percepción de los ciudadanos, al considerar que existe mucha corrupción en la Fuerza Pública 57,9\%; Tribunales $56,6 \%$ Defensoría $34,2 \%$ o algo de corrupción en la Defensoría 38,6\%; Fuerza Pública 29,7\%; Tribunales 29,2\%. Apenas un $8,2 \%$ de los ciudadanos conside- 
ran que no existe corrupción en las instancias de seguridad pública. Las distintas instituciones del poder público son corruptas, sin existir casi distinción entre ellas, faltando un elemento para la existencia de un buen gobierno.

Entre los efectos nocivos de la corrupción pueden mencionarse: malversación y dilapidación de recursos públicos, incumplimiento de los planes de gobierno, ineficiencia de la administración públi$\mathrm{ca}$, insuficiencia de inversión, falta de respuesta a las necesidades y problemas de la comunidad, clientelismo político, impunidad, desprestigio de las instituciones; en todas las áreas en las que interviene el Estado, sin permitir darle respuesta o satisfacer las necesidades humanas, mermando el desarrollo social y el nivel de vida de los ciudadanos (Diego, 2012).

Adicionalmente, la corrupción justifica en los ciudadanos la evasión de impuestos por considerar que los ingresos tributarios recaudados no se destinarán a la satisfacción de necesidades públicas sino que serán desviados para beneficio de pocos, por lo que se afecta la recaudación tributaria del país. En ese sentido, poco importa si el contribuyente ha sido víctima directamente de la corrupción o si solo es una percepción de las debilidades que tiene la democracia, igualmente afecta la moral tributaria (Castañeda, 2015). Otro resultado relevante fue que el $36,8 \%$ de los encuestados consideran que la corrupción política es la causante del contrabando que tiene entre otros efectos la evasión de impuestos.

La corrupción es un problema complejo por los diversos factores que la causan, para llegar a medidas anticorrupción efectivas es necesario el fortalecimiento de las instituciones, combinar medidas de arriba hacia abajo y promover la integridad como cultura (Boehm y Graf, 2009). Para disminuir la corrupción y mejorar el desempeño del gobierno que favorezca el Estado de derecho, es necesario:

"reformas al poder judicial y al sistema jurídico; institucionalidad y reformas a la administración pública, junto con la supervisión y participación de la sociedad civil y los medios de comunicación; la simplificación de las regulaciones económicas y la reforma tributaria, $y$ reformas financieras, del presupuesto y de las adquisiciones" (Kaufmann, 2014: 377)

El buen gobierno comprende más que la lucha contra la corrupción, dado que una mala administración pública rezaga el crecimiento y aumenta la pobreza; no obstante existe una relación directa entre gestión pública y corrupción, que agrava las desviaciones en la asignación de recursos, discrepancias en la distribución de ingresos y la riqueza afectando negativamente el nivel de vida; es decir, la corrupción además de quebrantar la confianza en el gobierno, tiene costos económicos (Mo, 2001). Las instituciones que son transparentes, rinden cuentas de manera eficiente, son maduras y luchan contra la corrupción, permiten que el Estado funcione y cumpla su misión (Acosta, 2015).

La corrupción es un problema complejo con diversas dimensiones, políticas, social, institucional, estructural, que exige soluciones igualmente estructural, pues la corrupción sería igual al abuso de poder, más impunidad, menos participación ciudadana (Sandoval, 2016).

En la lucha contra la corrupción es importante el enfoque que se adopte, mo- 
ral o de eficiencia, dependiendo de ello serán las estrategias, pues a veces estos dos enfoques no son compatibles por los efectos que pueden producir, en uno se busca eliminar la corrupción porque sí, constituyendo en algunos casos impedimentos para el funcionamiento de la administración pública, y en el otro que exista relaciones eficientes y eficaces entre el gobierno, el sector privado y los ciudadanos (Boehm y Graf, 2009; Cano, 2008). Dependiendo del tipo de corrupción será acertada la medida de control, si no preexiste resguardo a la integridad, cada funcionario y persona, redefine la ética de acuerdo a sus intereses (Anechiarico, 2010).

Existe una relación, entre más democracia menos corrupción. Es necesario incluir en la lucha contra la corrupción el fortalecimiento de las relaciones entre los representados y representantes a través del poder del voto, pues la corrupción indica la debilidad en los eslabones de la representación que fragmentan los procesos democráticos. La corrupción es una manera de exclusión que debilita los estímulos de la distribución del poder. Medidas contra la corrupción relacionadas con la apertura y transparencia, constituyen agentes ciudadanos, sea de manera individual o grupal, y medios de comunicación; las diversas normas jurídicas que regulan los derechos de información, participación, acceso a documentos públicos, dan más poder a los ciudadanos para intervenir en la supervisión de las actuaciones de la administración, dando lugar a denunciantes (Warren, 2005).

Por ser un delito requiere ser denunciada pero por su privacidad, secreto de la comisión del delito, es baja la denuncia, no reprochable socialmente e invasivo económicamente (Mannozzi, 2009). Entre las estrategias contra la corrupción se resalta las denuncias de los testigos, las víctimas y los socios. En el caso de las víctimas, es fundamental el papel del sistema judicial, si el mismo no es eficiente existiendo impunidad, no se logra disminuir la corrupción y se aumenta la desconfianza para con el gobierno (Boehm y Graf, 2009).

\section{Confianza institucional}

El papel de la confianza en el buen gobierno se hace fundamental al momento de considerarse como un elemento "explicativo de las actitudes hacia la democracia y, posteriormente, de los niveles de participación electoral. De lo anterior se desprende el estrecho vínculo entre confianza institucional, satisfacción con la democracia y legitimidad del sistema" (Morales, 2008: 163).

Siendo la democracia la representación de la voluntad del pueblo, el gobierno a través de instituciones como fuerza pública, tribunales, cárceles, contraloría, defensoría y entidades de índole nacional, departamental y municipal debe ser garante de credibilidad, accesibilidad y eficacia en sus actuaciones; sin embargo pareciera ser una constante la impopularidad, la corrupción y los malos manejos en las decisiones de los gobiernos tal como lo expresó el $90,7 \%$ de los encuestados quienes consideran que el sistema de justicia del Norte de Santander, no representa accesibilidad, imparcialidad y eficacia. Situación que genera desconfianza en el gobierno, incidiendo a 
la vez con el declive de los partidos políticos tradicionales, el surgimiento de partidos alternativos o a una posición radical de abstencionismo como lo señala Bartels (2000).

Siendo la confianza un indicador de percepción de los sentimientos ciudadanos sobre su sistema político (Moreno 2010), es coherente-con los anteriores resultados- que los entrevistados consideren que el sistema de justicia actual no representa los interés de la comunidad $92,1 \%$, siendo esto un agravante ya que legitima el actuar anómico y delictivo de la población pues un $51,3 \%$ de los encuestados creen que las personas particulares hacen justicia por sus propios medios, situación que resquebraja las relaciones colectivas y fundamenta aún más la desconfianza social e institucional.

Para Salcedo (2000: 159) la democracia debe darle al individuo la capacidad de "superar el aislamiento por medio de acuerdos que le permitan crear un poder común no tiránico", aislamiento que persistirá mientras la desconfianza social se traduzca en reacciones similares a las del gobierno que tanto se critica pero que se legitima con creencias como las de la mayoría de los encuestados, los cuales afirman con un $76,7 \%$ que la justicia solo responde a los intereses de las élites sociales, esta creencia se refleja en desinterés al momento de denunciar y de hacer presencia como veedores de políticas públicas, que permitan la minimización de las brechas sociales.

En cuanto a la confianza en las instituciones públicas, los encuestados respondieron nada $50,8 \%$; poco $28,9 \%$; algo $15,6 \%$; no sabe $2,0 \%$ y solo un $2,3 \%$ confía en ellas. Este panorama, permite ana- lizar la confianza a la luz de lo que Giddens (1997) denomina delito y bajo el cual se entiende la corrupción y es la existencia de múltiples factores como la anomia (la cual hace que se perciba impunidad para actuar libremente al momento de favorecer a otros), la asociación diferencial (la cual presenta ambientes sociales dicotómicos en los cuales se refuerza lo negativo como actos de aceptado), los valores innovadores los cuales resaltan la inequidad social y a la corrupción como una alternativa), la impunidad y el no denunciar son otros elementos, que refuerzan la persistencia de gobiernos que generan desconfianza en la ciudadanía.

Por su parte, Weber permite comprender la existencia de esta desconfianza cuando explica la evolución del gobierno democrático y deja ver como ésta obedece a la misma burocratización y capitalización del sistema electoral, el cual ha creado estructuras para gobernar política y administrativamente a tal punto, de involucrar sistemas económicos, académicos, militares, comunicativos con marcado interés, que han hecho posible la creación de algunos cargos a quienes "para permanecer necesitan de la confianza expresa de la mayoría... y por ende responder y rendir cuentas de modo exhaustivo" (Weber, 1922: 1095) mientras que los otros cargos, pagan favores que se han recibido al momento de las campañas electorales ya que ganar las mismas depende de "recursos políticos y económicos, ... en especial en aquellas en las que la lealtad de los votantes a los partidos políticos es débil" (Lenski et al, 1997:315).

Parece entonces, que se ha legitimado un círculo vicioso alrededor del go- 
bierno del cual los ciudadanos son conocedores y esto explica su apatía por lo político. Entre los encuestados se indagó sobre cuán interesados están en participar en lo público y el $19,1 \%$ manifestó que poco, mientras el $71,7 \%$ manifestó que nada. Correspondiente con esta percepción, se encontró que solo el $3,4 \%$ han participado alguna vez en un partido político.

Estas situaciones legitiman el actuar individualista que favorece a unos cuantos y a la vez sienta las bases para la desconfianza. Sabine (1937: 424) citando a Montesquieu, resalta la importancia de las reglas o normas de conducta que sirven para el buen funcionamiento social; y que sin embargo, con frecuencia son violadas por "la libertad de la voluntad y la defectuosa inteligencia de los hombres"; libertad y razonamientos que están girando sobre el bien y lucro personal.

La confianza es considerada como un conjunto de expectativas positivas sobre los demás o más específicamente, sobre las acciones de los demás. Tales expectativas se vuelven importantes cuando el individuo tiene que elegir un curso de acción a sabiendas de que su éxito depende de las acciones de otros; sin embargo, tiene que hacer esa elección antes de que pueda evaluar dichas acciones. La confianza, por tanto, tiene como características básicas: incertidumbre y reciprocidad (Foranda y Galindo, 2012: 52).

Desde el anterior concepto, la confianza es fundamental para que se dé un orden social ya que se requiere de acciones colectivas en las cuales todos participen y se unan esfuerzos que redunden en el bien común. Esta confianza es indis- pensable para lo que Putnam (2002) considera fundamental a la hora de construir capital social.

Para Torres et al, (2009) el análisis de la relación de confianza de un individuo con una institución, es diversa y se da según el tipo de servicio que se ejecute (para el caso analizado los pagos en línea, y los de mayor costo transaccional representan desconfianza) esto, desde el punto de vista del buen gobierno, evidencia también los grados de permisividad frente a la corrupción que manejan los ciudadanos y los cargos de los funcionarios públicos a quien perciban.

La confianza social representa un equilibrio entre las percepciones del individuo desde sus experiencias de vida y el contexto cultural en el cual ha sido formado, por ello Yáñez et al, (2006:19) proponen que "para construir hoy capital social, redes sociales y participación social, se necesita sin duda desarrollar habilidades sociales en la población", siempre que sean entendidas como inteligencia social lo cual los lleve a ser vigilantes no con ingenuidades de juicios positivos, sino con actitudes realistas y prudentes que les permitan establecer grados y asuntos específicos sobre los cuales confiar o desconfiar pues esto les brindará las herramientas de ciudadanía necesarias para la consolidación de una democracia que exija el buen gobierno.

Una cultura predominantemente democrática estará sujeta a las miradas locales así como sus ciudadanos, y una cultura predominantemente sustancial contendrá miradas locales y subjetivas de algunos ciudadanos, es decir será incluyente, con capacidad de ser llevada desde la base de las relaciones primarias 
las cuales transversalicen la familia, la comunicación y en general una comprensión de ciudadanía responsable de su actuar político (Almond y Verba, 1963).

\section{Conclusiones}

Analizadas las categorías ausencia de corrupción y confianza como elementos del buen gobierno en el Departamento Norte de Santander, se evidenció alta percepción sobre la existencia de corrupción en las instituciones públicas, lo cual incide en la credibilidad ciudadana. La misma es caracterizada por el incumplimiento de la norma, dependencia de los poderes públicos, falta de rendición de cuentas, poca transparencia, falta de control, carencias de recursos y poca participación ciudadana en los asuntos públicos, incidiendo en el funcionamiento del Estado y quebrantando la democracia.

La confianza como elemento de fortalecimiento del buen gobierno, debe sustentarse en las aptitudes de transparencia y actuación moral de los funcionarios públicos, quienes han de ver su rol como un ejercicio laboral que contribuye al desarrollo tanto de lo económico y lo democrático, como a la fundamentación de credibilidad de todo un sistema, que permita la inclusión y desvirtúe la creencia que la función pública es una plataforma para el lucro y el reconocimiento personal.

Los resultados sugieren que los ciudadanos tienen cierta responsabilidad en la existencia de corrupción en los organismos públicos, porque uno de los pilares fundamentales de un gobierno transparente es la participación ciudadana en la toma de decisiones, pero la gran parte de los encuestados, manifestaron una actitud indiferente al no desear participar en asuntos públicos; limitándose una de las estrategias de lucha contra la corrupción como es la participación ciudadana; que aunado a una ineficiente administración de justicia, fomenta hechos de corrupción no castigados.

Las instituciones (normas formales e informales) deben establecerse principalmente de arriba hacia abajo para que sean los organismos públicos, quienes propicien un clima de confianza por parte de los ciudadanos, así como modos de actuar ejemplarizantes. Los resultados de la investigación en el Norte de Santander, evidencian la percepción de corrupción y desconfianza en las instituciones públicas, lo que sugiere carencias para la existencia de un buen gobierno y limita la democracia participativa.

\section{Referencias bibliográficas}

Acosta Villada, Aurelio (2015), Las instituciones de fiscalización en México: su paso por la historia. Estudios Latinoamericanos, No. 31, pp. 111-134.

Aguilar, Felipe (2000), Moral pública en los procesos de buen gobierno. México: Plaza y Valdés Editores.

Albornoz, Neida; Gallardo, Henry; Mazuera Rina; Numa, Nahin; Orbegoso, Liliana; Ramírez, Carolina; Sánchez, Mónica y Torres, José (2016), Análisis del contrabando en el Norte de Santander, desde la percepción de los constructos de la institucionalidad. Cúcuta, Colombia: Universidad Simón Bolívar. Disponible en https:// issuu.com/usimon/docs/an lisis del contrabando en el nor

Almond, Gabriel y Verba, Sidney (1963), The Civic Culture: Political Attitudes 
and Democracy in Five Nations. United States of America: Princeton University Press.

Anechiarico, Frank (2010), La corrupción y el control de la corrupción como impedimentos para la competitividad económica. Gestión y política pública, Vol. 19, No. 2, pp. 239-261.

Aranda, Elviro (2013), Una reflexión sobre Transparencia y Buen Gobierno. Cuadernos Manuel Giménez Abad, No. 5, pp. 214-229.

Bartels, Larry (2000), Partisanship and Voting Behavior, 1952-1996. American Journal of Political Science, Vol. 44, No. 1, pp. 35-50.

Besançon, Marie (2003), Good governance rankings: The art of measurement. (Vol. 36). Cambridge, USA: World Peace Foundation.

Bhagwati, Jagdish y Gambari, Ibrahim (2005), Political will, not just aid, can lift Africa out of despair. Financial Times, pp. 13. Recuperado en http://www.columbia.edu/ jb38/papers/pdf/Political will in Africa.pdf Fecha de la consulta 09/07/2016.

Boehm, Frédéric y Graf Lambsdorff, Johann (2009), Corrupción y Anticorrupción: Una perspectiva neo-institucional. Revista de Economía Institucional, Vol. 11, No. 21, pp. 45-72.

Bonifacio, José (2006), La función pública en un buen gobierno. Revista Centroamericana de Administración Pública, No. 50, pp. 9-35.

Brower, Jorge (2015), Aportes epistemológicos para la comprensión de los conceptos de gobernabilidad y gobernanza. Revista Venezolana de Gerencia, Vol. 20, No. 72 , pp. 630-646.

Caddy, Joanne y Vergez, Christian (2003), Open Government: Fostering Dialogue with Civil Society. (OCDE) Publishing.
Campos, Jesús y Peña, Victor (2015), Implementación de políticas públicas de transparencia. El caso de las unidades de enlace en Coahuila, México. Revista Venezolana de Gerencia. Vol. 20, No. 72, pp. 612-629.

Cano, Luisa (2008), La participación ciudadana en las políticas públicas de lucha contra la corrupción: respondiendo a la lógica de gobernanza. Estudios Políticos, No. 33, pp. 147-177.

Carassale, Santiago (2013), Corrupción. Eunomía. Revista en Cultura de la Legalidad, No. 4, pp. 172-178.

Carbone, Maurizio (2010), The European Union, Good Governance and Aid Coordination. Third World Quarterly, Vol. 31, No. 1, pp. 13-29.

Castañeda Rodríguez, Víctor (2015), La moral tributaria en América Latina y la corrupción como uno de sus determinantes. Revista mexicana de ciencias políticas y sociales, Vol. 60 , No. 224, pp. 103-132.

Ciborra Claudio y Navarra Diego (2005), Good Governance, Development Theory, and Aid Policy: Risks and Challenges of E-Government in Jordan. Information Technology for Development, Vol. 11, No. 2, pp. 141-159.

CLAD (2006). Código Iberoamericano de Buen Gobierno. Centro Latinoamericano de Administración para el Desarrollo. Disponible en http://siare.clad.org/ siare/innotend/control/CodigolberoamericanodeBuenGobierno.pdf Fecha de la consulta: 05/10/2016.

CLAD (2009). Carta Iberoamericana de Participación Ciudadana. en la Gestión Pública, Aprobada por la XI Conferencia Iberoamericana de Ministros de Administración Pública y Reforma del Estado Lisboa, Portugal, 25 y 26 de junio de 2009. Adoptada por la XIX Cumbre Iberoamericana de Jefes de 
Estado y de Gobierno Estoril, Portugal, 30 de noviembre y 1 de diciembre de 2009.(Resolución No. 38 del" Plan de Acción de Lisboa").

Departamento Nacional de Planeación (DNP) (2016), Consultada el 28/05/2016. Recuperado de: https://www.dnp.gov. co/DNP/gestion/buen-gobierno/Paginas/Buen-Gobierno.aspx.

Diego Bautista, Oscar (2006), La ética y la corrupción en la política y la administración pública (Tesis de maestría). Universidad Internacional de Andalucía, Santa María de la Rábida, España.

Diego, Oscar (2012), El problema de la corrupción en América Latina y la incorporación de la ética para su solución. Espacios Públicos, Vol. 15, No. 35, pp. 48-62.

Doornbos, Martin (2001), 'Good Governance': The Rise and Decline of a Policy Metaphor?. The Journal of Development Studies. Vol. 37, No. 6, pp. 93108.

Doornbos, Martin (2006), 'Good Governance': The Metamorphoses of a Policy Metaphor. In Global Forces and State Restructuring ( $p p$. 73-92). Palgrave Macmillan UK.

Escuela Superior de Administración Pública (ESAP) (2007), Código de Buen Gobierno para la Escuela Superior de Administración Pública. Bogotá, Colombia.

Foronda-Robles, Concepción y Galindo-Pérez, Luis (2012), Argumentación relativa a la confianza territorial. Claves sobre capital social. Cuadernos de desarrollo rural, Vol. 9, No. 68, pp. $1-63$.

García, Jorge. (2000), Dinero y política. La cuadratura del círculo de la democracia en América Latina. Boletín Mexicano de Derecho Comparado, No. 99, pp. 1055-1083.
Giddens, Anthony (1997), Sociology. Madrid, España: Editorial Alianza.

Gobernación Norte de Santander. Decreto $N^{\circ}$ 000427 de fecha 29 de julio de 2008. Acto administrativo. Gaceta del Departamento. Colombia. Disponible en http://www.nortedesantander.gov.co /Portals/0/xBlog/uploads/2016/3/31/ 510acto DECRETO $000427 \mathrm{DEL}$ 29 DE JULIO DE 2008.pdf

Gómez, Alfonso (2006), Factores para un buen gobierno. Revista Reflexión Política, Vol. 8, No. 16, pp. 4-7.

Grindle, Merilee (2007), Good Enough Governance Revisited. Development Policy Review, Vol. 25, No. 5, pp. 553-574.

Hyden, Göran y Bratton Michael (1995), Governance and Politics in Africa. African Studies Review, Vol. 38, No. 1, pp. 156-159.

Kaufmann, Daniel (2014), Corrupción y reforma institucional: el poder de la evidencia empírica. Revista Perspectiva, Vol. 3, No. 2, pp. 367-387.

Larach, Catalina (2015), Transparencia y buen gobierno en España. Revista Digital de Derecho Administrativo, Vol. 13, pp. 255-268.

Leftwich, Adrian (1994), Governance, the State and the Politics of Development. Development and Change, Vol.25, No.2, pp.363-386.

Lenski, Gerhard; Nolan, Patrick and Lenski, Jean (1997), Human societies. An introduction to macrosociology. México: McGraw-Hill.

Mannozzi, Grazia (2009), Combatir a la corrupción. Un recorrido entre criminología y derecho penal. Boletín Mexicano de Derecho Comparado, No. 125, pp. 931-955.

Marín, Francisco (2004), La corrupción: ¿Un problema de sociedad y/o político? Frónesis, Vol. 11, No. 2, pp. 58-75. 
Mauro, Paolo (1995), Corruption and Growth. The Quarterly Journal of Economics, Vol. 110, No. 3, pp. 681-712.

Mo, Pak Hung (2001), Corruption and Economic Growth. Journal of Comparative Economics, Vol. 29, No. 1, pp. 66-79.

Montecinos, Egon (2012), Gestión municipal participativa: entre la nueva gerencia pública y la gobernanza democrática. Revista Venezolana de Gerencia (RVG), Año 17, № 58, pp. 345-364.

Morales, Eduviges; Núñez, Ingrid y Delfín, María Isabel (2006), Repensando desde el plano normativo la participación ciudadana en la gestión pública. Revista Venezolana de Gerencia (RVG), Año 11, № 35, pp. 453-470.

Morales, Mauricio (2008), Evaluando la confianza institucional en Chile: Una mirada desde los resultados LAPOP. Revista Ciencias Políticas (Santiago), Vol. 28, No. 2, pp.161-186.

Moro A. (2010). La confianza en las instituciones México en perspectiva comparada. Centro de Estudios Sociales y de Opinión Pública Cámara de Diputados / LXI Legislatura. México.

Nanda, Ved (2006), The "Good Governance" Concept Revisited. The ANNALS of the American academy of political and social science, Vol. 603, No. 1 , pp. 269-283.

Nonnel, Rosa (2006), Transparencia y buen gobierno. La rendición de cuentas en una sociedad avanzada. 2da edición. Barcelona, España: Icaria Editorial.

Oppenheimer, Nicky (2005), Enough handouts for Africa. International Herald Tribune, Disponible en https://journal.probeinternational.org/2005/07/12/enough-handouts-africa/.
Orrego, Claudio (2014), Corrupción y modernización del estado. New York, USA: Banco Interamericano de Desarrollo.

Panday, Pranab y Rabbani, Golam (2011) Good Governance at the Grass-roots Evidence from Union Parishads in Bangladesh. South Asian Survey, Vol. 18, No. 2, pp. 293-315.

Pezo, Eduardo (2012), Los principios de buen gobierno como principios rectores de la gestión y de las políticas del gobierno nacional y de los gobiernos regionales y locales en el marco de la descentralización. Derecho y Cambio Social, Vol. 9, No. 28, pp. 1-14.

Pollit, Christopher y Bouckaert, Geert (2000), Public Management Reform: A Comparative Analysis. Oxford: Oxford University Press.

Programa de las Naciones Unidas para el Desarrollo (PNUD) (2011), Documento de Política: Gobernabilidad para el Desarrollo Humano Sostenible. Disponible en: http://www.dse.de/zg/ $\mathrm{gg} / \mathrm{htmlenglish} / 4$ indephpaper 1.

htmv Fecha de la consulta 28/06/2016.

Putnam, Robert (2002), Solo en la bolera. Colapso y resurgimiento de la comunidad norteamericana. Barcelona: Galaxia Gutenberg.

Ramírez, José; Andreade, Jorge y Villarreal, Jesús (2005), Transparencia y combate a la corrupción. México: Secretaria de Educación Pública.

Sabine, George (1937), A History of political theory. Bogotá, Colombia: Fondo de cultura económica.

Salcedo, Marta (2000), Historia de las ideas políticas. Bogotá, Colombia: Ediciones doctrina y ley.

Sánchez, Javier (2015), Adela Cortina: el reto de la ética moral. BROCAR, Cuadernos de Investigación Histórica, $\mathrm{N}^{\circ}$ 39 , pp. 397-422. 
Sandoval, Irma (2016), Enfoque de la corrupción estructural: poder, impunidad y voz ciudadana. Revista Mexicana de Sociología. Vol. 78, No. 1, pp. 119152.

Soriano, Enrique (2014), El buen gobierno en la legislación española. En La regeneración del sistema: reflexiones en torno a la calidad democrática, el buen gobierno y la lucha contra la corrupción. Universidad Cardenal Herrera. Valencia, España, pp. 235-247.

The World Justice Project (2014), Rule of Law Index. Washington D.C., USA: World Bank.

Tordoff, William (2002), Government and politics in Africa. Bloomingtion, USA: Indiana University Press.

Tornos, Joaquín; Arroyo, Alfonso; Martínez, Mar y López, Juana (2012), Transparencia, rendición de cuentas y participación: una agenda común para la cohesión social y la gobernanza en América Latina (Vol. 6). En Colección de Estudios sobre Políticas Públicas Locales y Regionales de Cohesión Social. Barcelona, España: Programa URB-AL.

Torres, Eduardo; Manzur, Enrique; Olavarrieta, Sergio y Barra Cristóbal (2009), Análisis de la relación confianza-compromiso en la banca en internet. Revista Venezolana de Gerencia, Vol. 14 , No. 47, pp. 370-391.

Transparency International (2014), Corruption Perceptions Index 2014: Results. Disponible en https://www. transparency.org/cpi2014/results

Fecha de la consulta 05/10/2016.

Treisman, Daniel (2000), The causes of corruption: a cross-national study. Journal of Public Economics. Vol. 76, No. 3, pp. 399-457.

United Nations Development Programme (UNDP) (1995), Public Sector Management, Governance and Sustain- able Human Development. Nueva York, USA: Programa de Naciones Unidas para el Desarrollo.

United Nations Development Programme (UNDP) (1997), Governance for Sustainable Human Development: A UNDP Policy Document. New York, USA: United Nations Development Programme.

United Nations Development Programme (UNDP) (2014), Governance for Sustainable Development. Integrating Governance in the Post2015 Development Framework. Discussion Paper. New York. USA. Disponible en http://www.undp.org/content/dam/undp/library/Democratic\% 20Governance/Discussion-PaperGovernance-for-SustainableDevelopment.pdf Fecha de la consulta 09/07/2016

Villoria, Manuel (2013), El buen gobierno, entre la integridad institucional y la innovación democrática. Revista Democracia y Gobierno Local, No. 20, pp. 5-11.

Villoria, Manuel e Izquierdo, Agustín (2015), Ética Pública y Buen Gobierno. Madrid, España: Tecnos.

Warren, Mark (2005), La democracia contra la corrupción. Revista Mexicana de Ciencias Políticas y Sociales, Vol. 47, No. 193, pp. 109-141.

Weber, Max (1922), Wirtsschaft und gesellschaft. Grundriss der verstehenden soziologie. México: Editorial Fondo de Cultura Económica.

Weiss, Thomas (2000), Governance, good governance and global governance: conceptual and actual challenges. Third World Quarterly, Vol. 21, No 5, pp 795-814.

Woods, Ngaire (2000), The Challenge of Good Governance for the IMF and the World Bank Themselves. World Development, Vol. 28, No 5, pp. 823-841. 
World Bank (1992), Governance and Development, In Governance and Development. Washington, USA: World Bank.

Yáñez, Rodrigo; Ahumada, Luis; Cova, Félix (2006), Confianza y desconfianza: dos factores necesarios para el desarrollo de la confianza social. Revista Universitas Psychologica, Vol. 5, No. 1, pp. 9-20. 